\title{
Niveles de sobreviniencia y expectativas reduccionistas en biología
}

\author{
Levels of supervenience and reductionist \\ prospects in Biology
}

\author{
GUSTAVO CAPONI
}

Departamento de Filosofía

Universidad Federal de Santa Catarina // CNPq (Brasil)

\section{RESUMEN}

La existencia de propiedades biológicas sobrevinientes a las propiedades físicas no invalida el desarrollo de programas de investigación biológica de corte reduccionista como el de la Biología Molecular. La existencia de tales propiedades deja margen para el desarrollo de programas de investigación biológica que no asuman ese enfoque, pero no deslegitima los resultados obtenidos por programas que sí lo hacen. Sin embargo, reconocer que existen distintos grados de sobreviniencia, puede ayudarnos a determinar en dónde es dable esperar que la molecularización de la Biología genere lucros cognitivos efectivos y dónde es de temer lo contrario.

PALABRAS-CLAVE

FISICALISMO, BIOLOGIA MOLECULAR, REDUCCIONISMO, SOBREVINIENCIA

\begin{abstract}
The existence of biological properties that supervene on physical properties, do not invalidate the development of reductionist biological research programs such as Molecular Biology. The existence of such properties leaves room for the development of biological research programs that do not take that approach, but it does not annul results obtained by programs that follow that reductionist approach. However, recognizing that there are different degrees of supervenience,
\end{abstract}


can help us to determine where we should expect that the molecularization of Biology could generate cognitive profits and where we shouldn't expect that.

KEYWORDS

MOLECULAR BIOLOGY, PHYSICALISM, REDUCTIONISM, SUPERVENIENCE

El PRESENTE TRABAJO PERSIGUE DOS OBJETIVOS: sugerir que es posible establecer comparaciones de niveles, o de grados, de sobreviniencia; y apuntar la posibilidad de que dichas comparaciones den indicios sobre dónde, en qué frentes de investigación biológica, se puede suponer que la molecularización de la Biología sea capaz de generar progresos teóricamente significativos. O dicho de otro modo: reconocer, aunque sea de un modo vago e informal, que existen distintos grados de sobreviniencia, puede ayudarnos a determinar en qué dominios de las ciencias de la vida cabe esperar que la molecularización de la Biología fomente el planteamiento de problemas solubles relevantes, generando así lucros cognitivos efectivos y significativos, y en qué dominios es de temer que esa estrategia de investigación lleve a las ciencias de la vida por vías muertas en donde el rigor de los recursos metodológicos desplegados no se refleje en la relevancia de los resultados obtenidos.

Así, después de presentar el modo clásico de entender tanto la propia idea de sobreviniencia, como su relación con la cuestión del reduccionismo, esbozaré una versión gradual, o comparativa, de dicha noción. Y será a continuación que intentaré mostrar cómo es que ella puede permitirnos reformular la manera en la que hasta ahora ha sido defendida la legitimidad epistemológica de esos programas de investigación que responden a objetivos cognitivos ajenos al enfoque molecular de los fenómenos biológicos. Quiero mostrar que, en lugar de llevarnos a defender estos últimos programas impugnado los resultados que puedan obtenerse siguiendo la vía reduccionista, la idea de grados de sobreviniencia puede permitirnos justificar la expectativa, o la sospecha, de que, en algunos casos, esa última vía de investigación no va a llevarnos demasiado lejos, y no va a producir tales resultados.

Recordando el Curso de filosofía para científicos de Althusser, se puede decir que asumir o no una perspectiva reduccionista, es comprometerse o dejar de comprometerse con una línea de acción en el campo de la teoría -en el campo de la práctica teórica diría el propio Althusser-; y la idea de grados de sobreviniencia puede ayudarnos a evaluar la corrección (epistémica, claro) 
de ese compromiso. ${ }^{1}$ Pero no su corrección en un sentido abstracto, metafísico y a-histórico; sino su corrección en un sentido concreto y por referencia a una coyuntura particular en la historia de una ciencia. La idea de grados de sobreviniencia puede ayudarnos a determinar si, en un momento determinado del desarrollo de una disciplina biológica, comprometerse con un programa de investigación de corte reduccionista va a generar genuino progreso teórico, o sólo va a contribuir a la proliferación de datos pocos significativos y de experimentos muy costosos.

\section{UNA CARACTERIZACIÓN CLÁSICA Y GENERAL DE LA IDEA DE SOBREVINIENCIA}

La idea de sobreviniencia, vale apuntarlo, no tiene que ver únicamente con la relación existente entre propiedades biológicas y propiedades físicas. Ella tiene un sentido más general y puede aplicarse siempre que quiera aludirse a la relación entre propiedades o fenómenos que consideremos como más fundamentales, o básicos, y propiedades o fenómenos que consideremos necesariamente encarnados en esas propiedades o fenómenos más básicos, pero también como irreductibles a ellos. Se puede hablar de la sobreviniencia de las propiedades biológicas en relación a las propiedades físicas; pero también se puede hablar de la sobreviniencia de los fenómenos mentales en relación a los fenómenos neurofisiológicos. ${ }^{2} \mathrm{Y}$ es justamente para expresar más claramente esa tensión entre encarnación, o materialización, e irreductibilidad, que la noción de sobreviniencia fue amonedada y puesta en circulación.

Así, tomada en esa generalidad que permite recurrir a ella para hablar tanto de la relación existente entre propiedades físicas y propiedades biológicas, como de la relación entre fenómenos mentales y fenómenos cerebrales, pero también de cosas como la relación que puede haber entre propiedades o fenómenos atómicos, y propiedades o fenómenos moleculares, creo que la idea de sobreviniencia ha sido generalmente usada según esta regla: Una propiedad $X$ sobreviene a una configuración $Y$ de propiedades más básicas, si y solamente si, [1] X está presente siempre que Y esté presente, y [2] X puede estar presente aun cuando $Y$ no está presente. Así, cuando aplicada al caso específico de la relación entre propiedades biológicas y propiedades físicas, esa regla cobra la siguiente forma: Una propiedad biológica $B$ sobreviene a una configuración $F$ de propiedades físicas, si y solamente si, [1] B está presente siempre que $F$ esté presente, $y$ [2] B puede estar presente aun cuando $F$ no está presente. ${ }^{3}$

Pero, si el modo material en el que está siendo enunciada la noción de sobreviniencia nos incomoda, se puede buscar una traducción de la misma en el

$1 \quad$ L. Althusser 1974, p. 14.

2 E. Sober 1993, p. 73.

3 Al respecto, ver: E. Sober 1984, pp. 49-50; P. Lipton 2008, p. 117. 
modo formal de hablar. Hay una posible acepción de la idea de sobreviniencia bajo cuya consideración ella calificaría relaciones entre predicados antes que relaciones entre propiedades. Distinguiendo entre predicados propios de lo que consideremos una teoría o un discurso más básico y general, y predicados de lo que consideremos una teoría o un discurso menos básico y más especifico, esta acepción epistemológica, y no ontológica, del concepto de sobreviniencia podría ser enunciada de la siguiente manera: En un contexto teórico $T$, un predicado $P$ sobreviene a un predicado $R$ de carácter más básico, si y solamente si, [1] en $T, P$ debe predicarse de un individuo $X$ siempre que $R$ se predique de $X, y$ [2], aun en $T, P$ puede predicarse de otro individuo $Z$ aun cuando $R$ no pueda predicarse de $Z$.

Así, aplicado al caso específico de la relación entre predicados biológicos y predicados físicos, ese modo epistemológico de entender la idea sobreviniencia, es pasible de ser pensado como obedeciendo a esta regla: Un predicado biológico B sobreviene a un predicado físico $F$, si y solamente si, [1] B debe predicarse de un individuo $X$ siempre que $F$ se predique de $X, y$ [2], aun en $T$, $P$ puede predicarse de otro individuo $Z$ aunque $F$ no pueda predicarse de $Z$. La idea, en definitiva, es que no pueden establecerse diferencias biológicas si no existen diferencias físicas con las cuales correlacionarlas, pero que sí pueden establecerse semejanzas biológicas aun cuando no quepa, o no sea teóricamente relevante, establecer semejanzas físicas que justifiquen o le den soporte a esas semejanzas de índole específicamente biológica. No hay diferencia biológica sin diferencia física; pero si puede haber semejanza biológica sin semejanza física que la soporte. ${ }^{4}$

\section{SOBREVINIENCIA Y COMPROMISO REDUCCIONISTA}

Esa generalidad de la noción de sobreviniencia explica que ella haya podido ser usada tanto en el dominio de la Filosofía de la Mente como en el dominio de la Filosofía de la Biología; y siempre con intenciones análogas. La noción de sobreviniencia sirve, como acabo de decir, para caracterizar la relación entre, por una parte, procesos y estados mentales y, de otra parte, procesos y estados cerebrales. Pero ella tampoco deja de servir, como también acabo de decir, para examinar la relación existente entre, por un lado, propiedades y descripciones específicamente biológicas y, por otro lado, propiedades y descripciones moleculares de los seres vivos. Pero, tanto en un caso como en el otro la noción de sobreviniencia ha operado siempre en el mismo sentido: ${ }^{5}$ como un recurso para justificar lo que cabe describir como "un fisicalismo libre de compromi-

4 E. Sober 2010, p. 226.

5 A. Diéguez 2012, pp. 192-4. 
sos reduccionistas" ${ }^{6}$ o como un "fisicalismo no reductivo"? Y ahí ya hay una ambigüedad: porque 'estar libre de compromisos reduccionistas' puede simplemente significar que se está desobligado de tener que asumir una posición reduccionista, como también puede significar que se está libre de los errores del reduccionismo. En este último caso, la idea de sobreviniencia serviría para indicar que la propia ontología fisicalista es refractaria al reduccionismo.

Pero, si se acepta el tratamiento que Elliot Sober le dio a la relación existente entre sobreviniencia y reduccionismo en el caso específico de la Biología, ${ }^{8}$ se llega a la conclusión de que reconocer que existen propiedades biológicas que son sobrevinientes a las propiedades moleculares de los organismos, no implica que los programas de investigación biológica de corte reduccionista sean inviables o ilegítimos. La idea de sobreviniencia, conforme Sober la presenta, no serviría para poner en evidencia los errores del reduccionismo y para mostrarnos que el propio fisicalismo nos obliga a apartarnos de ellos. Aun sin invalidar los programas de investigación no-reduccionistas, el análisis que Sober deja abierta la posibilidad de que, a la larga, los programas de investigación que sí sean reduccionistas, se impongan y triunfen en todos los frentes de las ciencias de la vida. La aceptación de esa sobreviniencia, podemos decir, sólo nos exonera de la obligación de tener que comprometernos, aquí y ahora, con tales programas; y eso, sin ser poca cosa, es bastante menos que aquello que los anti-reduccionistas normalmente esperaban de esa idea. ${ }^{9}$ Ellos querían que ella sirviese para decretar, ab initio, la falencia del reduccionismo.

Es decir: aceptar que existen propiedades biológicas que son sobrevinientes a las propiedades moleculares de los organismos, nos permite asumir el fisicalismo sin por eso desestimar la legitimidad epistemológica, y la posible relevancia cognitiva, de programas de investigación pautados por preguntas que no sean pasibles de ser respondidas en términos de Biología Molecular. Pero eso no es lo mismo que postular un límite infranqueable para la búsqueda de explicaciones moleculares de los fenómenos orgánicos. En algún sentido, podríamos incluso pensar, todo se reduce a lo siguiente: el fisicalismo le promete el mundo a los programas de corte reduccionista; pero la idea de sobreviniencia parece relativizar esa promesa o tolerar el aplazamiento indefinido de su concreción, alentando así la apuesta en líneas de investigación que no sean reduccionistas. ${ }^{10}$ En definitiva, se podría también pensar, el recurso a la idea

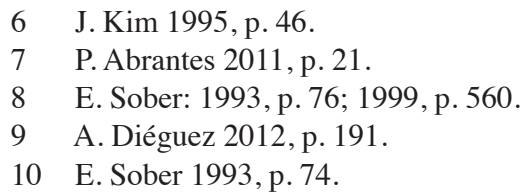


de sobreviniencia sólo nos brinda algo así como una salida negociada para la querella del reduccionismo.

Una salida que deja la solución de la cuestión librada a lo que el propio devenir de la Biología pueda decirnos sobre ella. Cosa que, por otra parte y vista desde cierto ángulo, no parece estar nada mal. Decidir a priori hasta dónde puede llegar un emprendimiento cognitivo en pleno desarrollo, y por dónde habrá de hacerlo, parece ser algo que escapa a las posibilidades de una reflexión epistemológica que no quiera arrogarse conocimientos transcientíficos y que, por eso, tampoco quiera verse enredada, como muchas veces ocurre, en divagaciones metafísicas sobre la naturaleza íntima de lo real. Será el propio futuro de la Biología el que nos dirá si el enfoque molecular puede desarrollarse y progresar al punto de tornar ociosos todos los demás modos de enfocar a los fenómenos biológicos; o si, en lugar de eso, continuará existiendo margen y motivo para el desarrollo de emprendimientos cognitivos que no obedezcan a ese punto de vista reduccionista.

De todos modos, y más allá de lo saludable y conveniente que pueda resultar la adopción de ese agnosticismo filosófico sobre cuestiones que compete a la ciencia resolver, yo pienso que la idea de sobreviniencia puede servirnos para algo más que para justificar esa salida negociada, o diferida, de la querella del reduccionismo. Ella, como dije, puede ayudarnos a la hora de juzgar posiciones y decisiones que deben tomarse actualmente en el desarrollo de las ciencias de la vida: posiciones y decisiones que tienen que ver con comprometerse, $o$ dejar de comprometerse, con agendas de investigación que sean, o no sean, de corte reduccionista. La idea de de sobreviniencia puede servirnos para juzgar la legitimidad que tienen las agendas de investigación que no siguen el vector epistemológico de la Biología Molecular; y ella también puede servirnos para estimar los lucros cognitivos que cabe esperar de esos programas de investigación que sí intenten seguir tales lineamientos.

Pero, para que la noción de sobreviniencia pueda operar de esa forma, hay que aceptar su uso comparativo asumiendo que las propiedades sobrevinientes a las propiedades físicas no son, todas ellas, sobrevinientes en un mismo grado. Hay que reconocer que la sobreviniencia, en general -de lo biológico a lo físico, de lo psíquico a lo cerebral, o de lo molecular a lo atómico-, no es una cuestión de todo o nada, no es una cuestión de 0 a $1 ;$ y reconociendo eso se puede pensar en establecer comparaciones de niveles o de grados de sobreviniencia. ${ }^{11}$ Siendo esos niveles de sobreviniencia los que habrán de darnos indicaciones sobre dónde, en que subdominio de las ciencias de la vida, es dable esperar que la molecularización de la Biología genere progreso teórico y en dónde es dable esperar que ocurra lo contrario. $\mathrm{O}$ dicho de otro modo: reconocer, aun cuando sea de

11 J. Yoshimi 2012, p.377 y ss. 
un modo vago e informal, que existen distintos grados de sobreviniencia, puede ayudarnos a determinar en dónde dable esperar que la molecularización de la Biología genere problemas solubles y en dónde es de temer que ella nos obligue a conformarnos con un cuestionario mucho menos ambicioso e interesante que aquél que se podía encarar asumiendo una perspectiva no-reduccionista.

\section{COMPARACIONES DE SOBREVINIENCIA Y GRADOS DE REDUCTIBILIDAD}

La idea de sobreviniencia, como vimos, conlleva dos elementos; el primero de los cuales puede ser formulado como si fuese la enunciación de un fisicalismo mínimo e innegociable que, a primera vista, parece obligarnos al reduccionismo explicativo: no hay diferencia sin diferencia física. Sin obligarnos a aceptar que las leyes o generalizaciones biológicas puedan ser un día trasformadas en teoremas de las leyes físicas, ese fisicalismo mínimo parece comprometernos con la idea de que todo objeto o fenómeno biológico es descriptible y analizable en términos físicos o químicos y que, consecuentemente, todo fenómenos biológico, a la larga, podrá ser explicado en esos mismos términos. ${ }^{12}$ Pero, el segundo elemento de la idea sobreviniencia, sin menoscabar ese fisicalismo mínimo, parece exonerarnos de las consecuencias metodológicas que este último parece imponernos: la presunción de que puede haber semejanza sin semejanza física deja margen para la adopción de perspectivas explicativas que no estén fundadas en conocimientos puramente físicos o químicos.

La idea de sobreviniencia, en definitiva, se juega en esa tensión: no hay diferencia sin diferencia física, pero puede haber semejanza sin semejanza física ${ }^{13}$ y las propiedades sobrevinientes son las que expresan esas semejanzas sin correlato físico. Son las propiedades sobrevinientes, para decirlo de otro modo, las que permiten delinear clases naturales que no tienen un referente físico acotado y definido; ${ }^{14}$ y esas clases naturales, a su vez, permiten reconocer invariantes causales explicativamente relevantes. ${ }^{15}$ Invariantes que justamente por el hecho de estar referidos a semejanzas no-físicas, serían difícilmente identificables por los ojos del físico. ${ }^{16}$ Soy consciente de que digo 'difícilmente identificables'; y no digo 'imposibles de ser identificados'.

Dos fenotipos posibles, en dos especies diferentes, pueden ser caracterizados como teniendo la misma eficacia darwiniana, aun cuando esas especies sean físicamente tan diferentes cuanto pueden serlo un ratón y una mariposa; ${ }^{17}$ y dos

12 S. Martínez 2011, pp.38-9.

13 A. Rosenberg 1985, p.113; J. Kim 1996, p.10; E. Sober 1984, pp.49-50.

14 A. Rosenberg \& D. McShea, 2008, p.114.

15 J. Woodward 2001, p.5.

16 J. Woodward 2008, p.260.

17 A. Rosenberg: 1985, pp.164-5. 
órganos pueden ser caracterizados como ojos aun cuando su estructura física, y el modo de registrar imágenes que uno y otro tengan, sean tan diferentes cuanto pueden serlo el ojo de un pulpo y el ojo de un halcón. Pero ya en esos dos ejemplos se insinúa una diferencia que no parece muy difícil de enunciar: ojo o visión son conceptos que parecen físicamente más acotados, físicamente más fácilmente delimitables, que eficacia darwiniana; aun cuando pueda pensarse que ese acotamiento, esa sinuosa línea que delimita la extensión física de lo que un ojo y la visión pueden ser, no sea, ni por aproximación, lo único que tengamos que conocer para poder entender qué es un ojo y qué es la visión.

Ver, ciertamente, es algo que puede ser hecho por estructuras que son físicamente muy diferentes; y lo mismo ocurre con digerir. Sin embargo, esa analogía funcional que se establece entre estructuras físicamente tan disímiles como pueden serlo el ojo de un pulpo y el ojo de un halcón, o entre el rumio de una vaca y el buche de una cucaracha, supone, pese a todo, un cierto grado de semejanza física que no puede ignorarse. Un ojo y un estomago son sistemas físicamente pluri-realizables; la propiedad de ser un ojo o de ser estomago es, en este sentido, sobreviniente a las propiedades físicas de las estructuras que pueden ser caracterizadas como ojos o estómagos. Pero, aun así, para que algo pueda ser considerado un ojo o un estómago, su funcionamiento normal debe tender a producir ciertos efectos, como registrar imágenes o contribuir en la descomposición de los alimentos ingeridos de forma tal que sus nutrientes puedan ser absorbidos y asimilados por el organismo; y esos efectos no dejan de tener una semejanza físicamente delimitable. Físicamente hablando, digerir y ver puede ser hecho de muchas formas; pero no cualquier proceso físico puede ser caracterizado como una digestión o como una visión. Hay algo, un conjunto de efectos físicos análogos [que quizá no sea, ni muy simple, ni muy importante, definir de forma precisa] que suponemos presente en todo lo que hemos de llamar 'digestión' o 'visión'.

Pero si ser un estómago o ser un ojo supone tener algún grado mínimo de semejanza física con otra cosa que también lo sea, a primera vista por lo menos, tener una eficacia darwiniana 1,5 no parece exigir nada de eso. Ese mismo índice de eficacia puede ser atribuido a una variante presente en una población de cucarachas urbanas y a una variante presente en una población de camarones marinos. Es decir, dos fenotipos tan diferentes cuanto pueden serlo el de una cucaracha y el de un camarón, sometidos a condiciones ambientales tan disímiles como las que cucarachas urbanas y camarones marinos deben enfrentar, pueden presentar una semejanza que no parece atada a ninguna semejanza física que sea relevante apuntar o conocer. Ahí parece haber un grado de sobreviniencia, o de pluri-realizabilidad, mucho mayor que en el caso de una caracterización 
funcional de una estructura como la que hacemos cuando decimos que algo es un ojo o un estomago.

No creo, sin embargo, que la realizabilidad variable, o la sobreviniencia, de un índice de eficacia darwiniana sea exacta y definitivamente absoluta; es decir: no creo que ella sea totalmente independiente de todo y cualquier substrato de semejanza física. O por lo menos no creo que la aceptación de esa independencia absoluta de cualquier substrato de semejanza física, sea una condición exigida para poder atribuirle un carácter sobreviniente a la eficacia darwiniana, o a cualquier otra propiedad o predicado. Walter Bock y Gerd von Wahlert, por ejemplo, propusieron definir el 'grado de adaptación' en términos de los recursos energéticos que un ser vivo necesita para realizar su ciclo vital en el nicho ecológico por él ocupado: a menor requerimiento energético, mayor adaptación; ${ }^{18}$ y ese modo de pensar, sobre todo en la medida en que se considera que la reproducción es parte del ciclo vital de un ser vivo, podría sugerir una posible delimitación física, ciertamente muy general, del concepto darwiniano de aptitud. Otra cosa distinta es saber cuánto es lo que realmente se aprendería con esa delimitación.

Creo, incluso, que nadie se atrevería a afirmar la existencia de una propiedad, atribuible a objetos empíricos, que quepa considerar como 'infinitamente sobreviniente' o 'infinitamente pluri-realizable'. Hasta me permito a decir que, estrictamente hablando, tampoco existen propiedades que sean nulamente sobrevinientes. Creo, en todo caso, que existen propiedades cuya sobreviniencia es despreciable por aproximarse indefinidamente a cero; y que, en el otro extremo de la escala, existen propiedades cuya pluri-realizabilidad es tan grande que su delimitación difícilmente llegue a ser cognitivamente relevante. La delimitación física de lo que es un líder carismático no parece interesarle a ningún sociólogo o historiador. Y esa referencia a la relevancia cognitiva también es importante para aclarar la diferencia existente entre decir que algo es un ojo y atribuirle a un fenotipo un cierto índice de eficacia darwiniana. Es que, contrariamente a lo que ocurre con la atribución de una propiedad funcional como ser un estomago, la atribución de un índice particular de eficacia darwiniana no requiere de un conocimiento, ni siquiera vago o aproximado, del conjunto de propiedades físicas compartidas por todo y cualquier fenotipo al cual quepa atribuirle ese índice de eficacia.

No es que esas propiedades físicas definitivamente no existan; sino que el conocimiento de las mismas, resulta mucho menos relevante que en el caso de una identificación anátomo-funcional. Y ahí, en la consideración simultánea del aspecto ontológico y del aspecto epistemológico de la cuestión, está la clave para darle una forma un poco más clara a la idea de niveles o grados de sobre-

18 W. Bock \& G. Wahlert [1965] 1998, pp.145-6. 
viniencia que aquí estoy procurando explicitar: dados dos sistemas u objetos, cuando menor sea el grado de semejanza física entre ellos del que depende la correcta atribución de una propiedad común a ambos, y cuando menos relevante sea un conocimiento preciso de esa semejanza para justificar tal atribución, mayor es el grado de sobreviniencia de dicha propiedad. Aunque otra forma de definir esa misma idea también puede ser la siguiente: Dados dos sistemas u objetos, cuanto menos delimitado o especificado esté el conjunto de predicados físicos del que depende la correcta atribución a ambos de un predicado biológico, más sobreviniente será ese predicado.

"Ser un tubo digestivo" o "ser un organismo fotosintético" son, en este sentido, propiedades menos sobrevinientes que "ser una homoplasia" o "ser una adaptación"; y estas dos últimas propiedades son ciertamente más sobrevinientes, o más pluri-realizables, que "ser una característica mimética" o que "ser un predador", aun cuando estas últimas lo sean en menor grado que las dos primeras. Y esas comparaciones de grados de sobreviniencia se mantienen idénticas si, en vez de hablar de propiedades hablamos de predicados. Pero, más que esa dualidad, lo que aquí quiero destacar es la relevancia, o la utilidad, que, según he dicho, esa idea de niveles o grados de sobreviniencia puede tener para un mejor planteamiento de la cuestión del reduccionismo. Los niveles de sobreviniencia -lo digo sin pensar que estoy haciendo una gran revelacióndenuncian grados de reductibilidad.

Conforme lo apunté al inicio, aceptar que existen propiedades biológicas sobrevinientes a las propiedades físicas no conlleva una condena de las expectativas reduccionistas que podrían estar implicadas en el proyecto de una molecularización integral de la Biología. A lo que esa aceptación conduce, en todo caso y como también ya fue dicho, es a un reconocimiento de la legitimidad de los programas de investigación no-reduccionistas que se desarrollan en Biología. Comprometerse con estos últimos programas, podemos quedarnos tranquilos, no implica ir en contra del fisicalismo; aunque eso no quiera decir, ni suponga creer, que en los fenómenos biológicos exista algo que sea intrínseca e irremediablemente inabordable desde un enfoque puramente molecular. Es obvio, sin embargo, que existen aspectos o dominios de los fenómenos biológicos que se han mostrado menos permeables que otros a ese enfoque; y es eso lo que, según me parece, puede ser entendido apelando a la idea de niveles o grados de sobreviniencia.

La idea es ésta: aquellos aspectos de los fenómenos biológicos cuya caracterización envuelve propiedades, o predicados, menos sobrevinientes, se prestan más a los desarrollos reduccionistas que aquellos aspectos de dichos fenómenos que pueden ser caracterizados haciendo referencia a propiedades, o predicados, más sobrevinientes. La Neurofisiología sería un buen ejemplo de lo primero y la 
Etología Cognitiva ${ }^{19}$ lo sería de lo segundo. Por eso, cuanto mayor es el grado de sobreviniencia de las propiedades estudiadas, más libertad tenemos para no comprometernos en tentativas de explicar los fenómenos a ellas asociados que obedezcan a una perspectiva reduccionista; y más inciertos son los lucros cognitivos de ese compromiso. Propiedades altamente sobrevinientes son difíciles de encarar desde una perspectiva reduccionista y es posible que el esfuerzo, no pague; y si el reduccionismo no paga, vale intentar por otra vía. Lamarck, por ejemplo, llegó a creer que podía explicar el origen de toda la diversidad de formas vivas conocidas, recurriendo a una legalidad puramente física; Darwin intentó una vía totalmente diferente, y todos sabemos que le fue mucho mejor.

\section{CONSIDERACIONES FINALES}

En realidad, lo que he dicho no sólo se aplica a la Biología Molecular. Me he referido a ella simplemente porque es por ahí que, en general, se piensa que debería consumarse la traducción de toda la Biología en un discurso perfectamente congruente con el discurso de la Física. ${ }^{20}$ Pero la idea de niveles de sobreviniencia podría también servir para evaluar las promesas de la Ecología Metabólica, ${ }^{21}$ que tampoco dejan de ser explícitamente reduccionistas, ${ }^{22}$ y ciertamente también sería útil para juzgar hasta dónde se podría llegar intentando aproximaciones cripto-reduccionistas entre Biología Evolucionaria y Física, como esas propuestas alguna vez por Ilya Prigogine ${ }^{23} \mathrm{y}$, un poco más recientemente, por Brian Goodwin. ${ }^{24}$ Pero, además de eso, la vía de análisis que aquí esbocé tiene consecuencias que van más allá de las cuestiones planteadas por la posible homogenización del saber biológico y el saber físico-químico. Las propias ambiciones imperialistas de la Biología pueden ser evaluadas en base a la idea de niveles de sobreviniencia.

Intentar explicar neurofisiológicamente una reacción de pánico promete lucros cognitivos más inmediatos y significativos que intentar explicar neurofisiológicamente la opción por un candidato presidencial de determinado votante. Nadie duda de que esa opción responde, en última instancia, a un fenómeno neurofisiológico que hasta puede ser molecularmente explicable: todos somos fisicalistas. Pero si queremos tener un conocimiento significativo sobre por qué

19 Sobre las peculiaridades epistemológicas de la Etología Cognitiva, véase: C. Allen \& M. Bekoff 1997.

20 S. Sarkar 1998, pp.136-7; A. Rosenberg 2006, pp.56-7

$21 \mathrm{Al}$ respecto, ver: J. Brown et al 2004.

22 Al respecto, véase: F. Bouchard 2007, pp.74-6; G. Folguera \& F. Di Pasquio 2011, pp.144-5.

23 I. Prigogine [1977]1983. Al respecto del cripto-reduccionismo de Prigogine, ver: O. Lombardi 1999, p.137.

24 B. Goodwin 1998, p.209. 
las personas votan como votan, un conocimiento que hasta nos permita anticipar ese voto e incluso manipularlo con la propaganda y la mentira, es muy posible que la neurofisiología nos diga mucho menos que una investigación sociológica clásica, basada en entrevistas y no en imágenes obtenidas por Pet Scan. Con relación a las propiedades neurofisiológicas, "ser conservador" o "ser de izquierda" son propiedades mucho más sobrevinientes que "estar en pánico" o "estar estresado". Por eso nadie, sobre todo ningún sociólogo, se toma muy en serio la posibilidad de explicar las posiciones políticas en términos neurofisiológicos.

\section{REFERENCIAS BIBLIOGRÁFICAS}

ABRANTES, P. 2011: «A esfera do mental: filosofia, ciência e senso comum», en C. Chitolina; J. Pereira; J. Dias; L. Montagna; R. Pinto (eds.), A natureza da mente, Maringá: Humanitas Vivens, pp.17-45.

ALLEN, C. \& BEKOFF, M. 1997: Species of mind: the philosophy and biology of Cognitive Ethology, Cambridge: MIT Press.

ALTHUSSER, L. 1974: Philosophie et philosophie spontanée des savants [introduction au Cours de philosophie pour scientifiques -1967-], Paris: Maspero.

BOCK, W. \& WAHLERT, G. [1965]1998: «Adaptation and the form-function complex», en C. Allen; M. Bekoff; G. Lauder (eds.), Nature's purpose: analyses of function and design in Biology. Cambridge: MIT Press, pp.117-168.

BOUCHARD, F. 2007: «Moving beyond the influence of Molecular Genetics on the debate about reductionism in Philosophy of Biology»", en A. Fagot-Largeault; S. Rahman; J. Torres (eds.), The influence of Genetics on contemporary thinking, Dordrecht: Springer, pp.63-80.

BROWN, J.; GILLOOLY, J.; ALLEN, A.; SAVAGE, M.; WEST, G. 2004: «Toward a metabolic theory of Ecology », Ecology, 85(7), pp.1771-1789.

DIÉGUEZ, A. 2012. La vida bajo escrutinio, Barcelona: Buridán.

FOLGUERA, G. \& DI PASQUIO F. 2011: «Jerarquías ecológicas: intentos reductivos de la Fisiología a través de la Macroecología», Ludus Vitalis, 19(36), pp.137-151.

GOODWIN, B. 1998: Las manchas del leopardo, Barcelona: Tusquets.

KIM, J. 1995: «El mito del materialismo no reduccionista», Análisis Filosófico, 15, pp. 35-56.

KIM, J. 1996: Philosophy of Mind, Colorado: Westview Press.

LIPTON, P. 2008: «CP Laws, reduction and explanatory pluralism», en J. Hohwy \& J. Kallestrup (eds.), Being Reduced, Oxford: Oxford University Press, pp.115-125.

LOMBARDI, O. 1999: «Prigogine y la reducción en ciencias», Cadernos de História e Filosofia da Ciência, S.3, 9(1-2), pp.123-145. 
MARTÍNEZ, S. 2011: «Reduccionismo em Biologia», en P. Abrantes (ed.), Filosofia da Biologia, Porto Alegre: Artmed, pp.37-52.

PRIGOGINE, I. [1977]1983: «La evolución de la complejidad y las leyes de la naturaleza», en I. Prigogine, ¿Tan sólo una ilusión?, Barcelona: Tusquets, pp.221304.

ROSENBERG, A. 1985: The structure of biological science, Cambridge: Cambridge University Press.

ROSENBERG, A. \& McSHEA, D. 2008: Philosophy of Biology, London: Routledge.

SARKAR, S. 1998: Genetics and reductionism, Cambridge: Cambridge University Press.

SOBER, E. 1984: The nature of selection. Chicago: The University of Chicago Press. , 1993: Philosophy of Biology, Oxford: Oxford University Press. , 1999: «The multiple realizability argument against reductionism», Philosophy of Science, 66, pp.542-564.

, 2010: «Learning from functionalism: prospect for strong artificial life», en M. Bedau \& C. Cleland (eds.), The nature of life: classical and contemporary perspectives from Philosophy and Science. Cambridge: Cambridge University Press, pp.225-235.

WOODWARD, J. 2001: «Law and explanation in Biology: invariance is the kind of stability that matters», Philosophy of Science, 68, pp.1-20.

, 2008: «Mental causation and neutral mechanisms», en J. Hohwy \& J. Kallestrup (eds.), Being reduced, Oxford: Oxford University Press, pp.218-262.

YOSHIMI, J. 2012: «Supervenience, dynamical systems theory and non reductive physicalism», British Journal for the Philosophy of Science, 63, pp.373-398.

Gustavo Caponi es profesor del Departamento de Filosofía de la Universidad Federal de Santa Catarina e investigador del Consejo Nacional de Investigaciones del Brasil $(C N P q)$.

Líneas de Investigación:

Filosofía de la Biología, Historia de la Biología

Publicaciones recientes:

(2012) Função e desenho na biologia contemporânea, Associação Scientiae Studia // Editora 34: São Paulo, 2012.

(2011) La segunda agenda darwiniana: contribución preliminar a una historia del programa adaptacionista, Centro de Estudios Filosóficos, Políticos y Sociales Vicente Lombardo: México, 2011.

Dirección electrónica: gustavoandrescaponi@gmail.com 